\title{
Pinelliae Rhizoma Praeparatum Cum Alumine Extract: Sedative and Hypnotic Effects in Mice and Component Compounds
}

\author{
Sisi Lin $\mathbb{D}^{1}$, Bo Nie $\mathbb{D}^{2}$, Ke Song $\mathbb{D}^{2}$, Ren Ye $\mathbb{D}^{1}$, and Zhengzhong Yuan $\mathbb{D}^{1}$ \\ ${ }^{1}$ Department of Traditional Chinese Medicine, The First Affiliated Hospital of Wenzhou Medical University, Wenzhou 325000, China \\ ${ }^{2}$ Key Laboratory of Chinese Internal Medicine of Ministry of Education and Beijing, Dongzhimen Hospital, \\ Beijing University of Chinese Medicine, Beijing 100700, China
}

Correspondence should be addressed to Ren Ye; yeren601@163.com and Zhengzhong Yuan; wzyzz2008@126.com

Received 4 December 2018; Revised 25 March 2019; Accepted 8 April 2019; Published 28 April 2019

Academic Editor: Sushil K. Jha

Copyright (c) 2019 Sisi Lin et al. This is an open access article distributed under the Creative Commons Attribution License, which permits unrestricted use, distribution, and reproduction in any medium, provided the original work is properly cited.

Pinelliae Rhizoma Praeparatum Cum Alumine (PRPCA) is useful for eliminating dampness and phlegm in clinical settings, targeting the main mechanisms of insomnia as defined in traditional Chinese medicine. However, little is known regarding the sedative and hypnotic effects of PRPCA. In the present study, we examined the sedative effects of PRPCA via a locomotor activity test and aimed to determine the most appropriate concentration of PRPCA for achieving these effects. The strongest sedative effects were observed at a PRPCA concentration of $0.45 \mathrm{~g} / \mathrm{ml}$. In addition, we investigated the hypnotic effects of PRPCA and its role in promoting sleep via sleep monitoring and vigilance state analysis. PRPCA increased rapid eye movement (REM) sleep and non-REM (NREM) sleep while decreasing wakefulness. In addition, PRPCA decreased the number of bouts of wakefulness (16-32 s and 32-64 s) and increased the number of bouts of NREM sleep (128-256 s). Furthermore, we identified a total of 32 component compounds via chromatography and mass spectrometry. Hence, the current work provides valuable information regarding the sedative and hypnotic effects of PRPCA and its regulatory mechanisms in promoting sleep.

\section{Introduction}

Insomnia is a common and significant public health problem worldwide [1], exerting substantial effects on society and the economy due to workplace absenteeism and accidents and decreases in productivity $[2,3]$. The treatment of insomnia can be complex and time-consuming for both patients and providers. Effective treatment requires appropriate diagnosis, cognitive behavioral therapy for insomnia (CBT-I), and pharmacological treatment. Although CBT-I is regarded as a first-line treatment option [4], there are still several barriers that limit its use, including a lack of qualified clinicians, geographical remoteness, stigma associated with receiving psychological services, perceived costs, and the dissemination of inadequate information among patients and providers [5-7]. Considering their side-effects, pharmacological agents should be prescribed with caution and are recommended for short-term use only. Given these limitations, researchers worldwide have begun to focus on developing safer, more effective, and more economical treatment strategies for insomnia.

In traditional Chinese medicine, phlegm stagnation and disconnection between Yin and Yang are the main causes of insomnia. Pinelliae Rhizoma (PR) can treat insomnia by eliminating dampness and phlegm and connecting Yin and Yang [8]; it also reduces the incidence of adverse reactions and alleviates vomiting [9, page 119]. The first recorded use of PR for the treatment of insomnia is described in HuangdiNeijing, a classic work in traditional Chinese medicine. Song et al. [10] noted that there are 177 prescriptions for insomnia described in the HuangdiNeijing and Chinese medical literature published no later than 1911, including 159 prescription drugs. Among them, PR is listed as the sixth most utilized drug. However, considering the toxicity of its raw form, PR is usually processed in order to enhance its efficacy and reduce toxicity. In our recent study [11], we observed that 
the Pinellia Ternata (Thunb.)Makino preparation-which is prepared by processing raw $\mathrm{PR}$ with licorice, lime, and alum as adjuvant materials-promotes sleep and sedation in mice by increasing REM sleep. PRPCA is another preparation of $\mathrm{PR}$ with a simpler preparation process. In this preparation, the quality of the drug is affected by fewer factors, and PRPCA is relatively easier to obtain with better quality control. PRPCA is processed after soaking PR in Alumine solution, which is an astringent that effectively eliminates dampness. This procedure reduces the toxicity of the drug as well as irritation, enhancing its ability to eliminate dampness and phlegm. According to the Pharmacopoeia of the People's Republic of China (2015), PRPCA exhibits lower toxicity than raw PR [9, page 120]. Tao et al. [12] compared the toxicity and irritating effects of raw PR, PR processed with ethanol, and PRPCA and reported that PR processed with ethanol and PRPCA significantly reduced irritation in the rabbit conjunctiva and prostaglandin E2 (PGE2) content (an inflammatory mediator) in peritoneal exudates from rats. Although this study highlighted the safety of PRPCA, little is known regarding its sedative and hypnotic effects.

\section{Materials and Methods}

2.1. Experimental Design. The present study included three parts: (a) investigation of the sedative effects of PRPCA in mice and the proper dose of PRPCA; (b) investigation of the hypnotic effects of PRPCA in mice and its role in promoting sleep; (c) identification of the chemical components of PRPCA.

2.2. Drugs and Reagents. PRPCA is a type of PR preparation (i.e., dried tuber of Pinellia Ternate (Thunb.) Breit.) that is processed with alumine as an adjuvant material. PRPCA granules (batch number: 1510002S) were purchased from China Resources Sanjiu (Beijing) Pharmaceutical Co., Ltd (Beijing, China), and identified by Xiaochen Chen, the deputy director of the TCM pharmacy at the First Affiliated Hospital of Wenzhou Medical University in Zhejiang, China. Diazepam (DZP, batch number: AH120701) was purchased from Shanghai XudongHaipu Pharmaceutical Co., Ltd. (Shanghai, China) for use as a positive control. Sterile water for injection (SWFI, batch number: M11073107) was purchased from Fuzhou Haiwang Pharmaceutical Co., Ltd. (Fujian, China) for use as a placebo and solvent. High-performance liquid chromatography- (HPLC-) grade acetonitrile was purchased from J.T.Baker Company (J.T.Baker, USA). Ultrapure water produced using a MilliQ water purification system (18.2 $\mathrm{M} \Omega$, Millipore, Bedford, MA, USA) was used in all aqueous solutions.

2.3. Drug Preparation and Extraction. Each bag of PRPCA contained $0.5 \mathrm{~g}$ of PRPCA granules, which is equivalent to $6 \mathrm{~g}$ of raw PRPCA. In our preliminary experiment [13], 4.5 bags, 9 bags, and 18 bags of PRPCA granules were dissolved in SWFI and titrated to $60 \mathrm{ml}$ to yield concentrations of $0.45 \mathrm{~g} / \mathrm{ml}$, $0.9 \mathrm{~g} / \mathrm{ml}$, and $1.8 \mathrm{~g} / \mathrm{ml}$ (equal to treatment doses of $9 \mathrm{~g} / \mathrm{kg}$, $18 \mathrm{~g} / \mathrm{kg}$, and $36 \mathrm{~g} / \mathrm{kg}$ when $0.02 \mathrm{ml} / \mathrm{g}$ was intragastrically administered to mice), respectively. The prepared solutions were stored in a refrigerator with a temperature below $4^{\circ} \mathrm{C}$. One tablet of DZP was dissolved in $25 \mathrm{ml}$ of SWFI to obtain $0.1 \mathrm{mg} / \mathrm{ml}$ of liquid DZP.

PRPCA granules were crushed and soaked overnight in a cold acetonitrile: water solution (3:1, v/v), following which they were subjected to $45 \mathrm{~min}$ of ultrasonification for extraction. The extract was centrifuged at 10,000 r/min for $10 \mathrm{~min}$. The supernatant was filtered through a 0.22 $\mu \mathrm{m}$ microporous membrane and stored at $-80^{\circ} \mathrm{C}$ for liquid chromatography-mass spectrometry (LC-MS) analyses.

2.4. Animals and Grouping. Male SPF C57BL/6J mice weighing 20-25g (10-12 weeks old) were used for the present study. Mice were supplied by the Laboratory Animal Centre at the Chinese Academy of Sciences (Shanghai, China). The animals were individually housed at a constant temperature $\left(22 \pm 0.5^{\circ} \mathrm{C}\right)$ and relative humidity of $60 \% \pm 2 \%$. Animals were housed under a 100-lux 12-hour light/dark cycle (lights on 7:00 AM to 7:00 PM) and were provided ad libitum access to food and water. This study was performed in accordance with the recommendations of the National Institutes of Health Guide for the Care and Use of Laboratory Animals and the experimental laboratory animal ethics committee of the Laboratory Animal Center at Whenzhou Medical University. The protocol was approved by the experimental laboratory animal ethics committee of the Laboratory Animal Center at Whenzhou Medical University (wydw 2016-026).

For the locomotor activity test, all 30 mice were randomly divided into the following five groups: (1) the placebo group, in which mice were intragastrically administered SWFI; (2) the positive control group, in which mice were intragastrically administered DZP at $0.1 \mathrm{mg} / \mathrm{ml}$; (3) the PRPCA 1 group, in which mice were intragastrically administered PRPCA at $0.45 \mathrm{~g} / \mathrm{ml}$; (4) the PRPCA 2 group, in which mice were intragastrically administered PRPCA at $0.9 \mathrm{~g} / \mathrm{ml}$; and (5) the PRPCA 3 group, in which mice were intragastrically administered PRPCA at $1.8 \mathrm{~g} / \mathrm{ml}$.

For sleep monitoring and vigilance state analyses, all 18 mice were randomly divided into the following three groups: (1) the placebo group: mice were intragastrically administered SWFI; (2) the positive control group: mice were intragastrically administered DZP at $0.1 \mathrm{mg} / \mathrm{ml}$; and (3) the PRPCA group: mice were intragastrically administered PRPCA at $0.45 \mathrm{~g} / \mathrm{ml}$. All drug volumes were calculated based on the weights of the mice, as follows: mouse weight $\times 0.02$.

2.5. Locomotor Activity Test. To investigate the sedative effects of different concentrations of PRPCA and determine the most effective concentration, we assessed locomotor activity in experimental mice.

Each mouse was housed in an individual recording container $(28 \mathrm{~cm} \times 16.5 \mathrm{~cm} \times 13 \mathrm{~cm})$ with a passive infrared sensor (Biotex, Kyoto, Japan) placed $17.5 \mathrm{~cm}$ above the floor of the container, as previously reported [14]. All detectors were connected to the computer to record mouse movement during the test. Prior to behavioral recording, all mice were habituated to the container for $24 \mathrm{~h}$. Then, the drugs were 
intragastrically administered to mice at 7:00 PM for 14 days. Locomotor activity was continuously recorded during the 14day period, and the data were fed back to the computer every $5 \mathrm{~min}$. Locomotor activity for each $24 \mathrm{~h}$ period was obtained by summing the corresponding records from 7:00 PM on one day to 7:00 PM on the following day.

2.6. Sleep Monitoring and Vigilance State Analyses. Under $5 \%$ chloral hydrate anesthesia $(400 \mathrm{mg} / \mathrm{kg}$, i.p.), mice were simultaneously implanted with electrodes for polysomnographic electroencephalography (EEG) and electromyography (EMG) recording [15]. Briefly, two stainless steel screws ( $1 \mathrm{~mm}$ in diameter) were inserted through the skull above the cortex (anteroposterior, $+1.0 \mathrm{~mm}$; left-right, $-1.5 \mathrm{~mm}$ from bregma or lambda) [16] to monitor EEG signals, and two insulated stainless steel screws were placed bilaterally into both trapezius muscles to function as EMG electrodes. All electrodes were attached to a microconnector and fixed to the skull using dental cement, following which the wounds were closed.

A slip-ring was designed to ensure that the behavioral movement of the mice would not be restricted during EEG and EMG recording [17]. After a 7-day recovery period, mice were individually housed in transparent barrels and habituated to the recording cable for $24 \mathrm{~h}$ prior to sleep monitoring. Then, the drugs were intragastrically administered to mice at 7:00 PM for 14 continuous days. After this 14-day period, sleep-wakefulness states were monitored for $24 \mathrm{~h}$.

The EEG/EMG signals were amplified, filtered (EEG: 0.5-30 Hz; EMG: 20-200 Hz), digitized at a sampling rate of $128 \mathrm{~Hz}$, and recorded using SleepSign software (Kissei Comtec, Nagano, Japan), as described elsewhere [18, 19]. Vigilance was then automatically classified offline under $4 \mathrm{~s}$ epochs into wakefulness, rapid eye movement (REM) sleep, and non-REM sleep (NREM) using SleepSign software, in accordance with standard criteria [20]. Wakefulness is characterized by low-amplitude, high-frequency EEG activity, and high EMG activity. REM sleep is characterized by lowamplitude, high-frequency EEG activity, and an absence of EMG activity. The presence of EEG theta activity $(6-9 \mathrm{~Hz})$ can be used to confirm this state. NREM sleep is commonly characterized by high-amplitude EEG activity and lowvoltage EMG activity. The presence of high delta activity on EEG $(0.65-4 \mathrm{~Hz})$ is also used to define this state. Finally, the defined sleep-wake stages were visually examined and corrected if necessary.

2.7. Chromatography and Mass Spectrometry. The samples were analyzed using a Waters Acquity ultra-performance liquid chromatography system (UPLC, Waters Corporation, Milford, MA, USA) equipped with an ACQUITY UPLC HESS C18 column $(2.1 \mathrm{~mm} \times 100 \mathrm{~mm}, 1.8 \mu \mathrm{m})$. The UPLC system consisted of a vacuum degasser, a binary pump, an autosampler, a column heater, and a diode array detector (DAD) coupled to a quadrupole time-of-flight mass spectrometry (QTOF) analyzer in a SYNAPT G1 system (Waters Corporation) and equipped with an electrospray ionization (ESI) interface.
TABLE 1: Impact of time and group on locomotor activity in mice.

\begin{tabular}{lcc}
\hline & $\mathrm{F}$ & $\mathrm{P}$ \\
\hline Time & 2.133 & 0.088 \\
Group & 4.267 & $\leq 0.001$ \\
Time*Group & 6.242 & 0.004 \\
\hline
\end{tabular}

Water served as mobile phase A, while acetonitrile served as phase $\mathrm{B}$. The temperature of the column was set to $40^{\circ} \mathrm{C}$. The elution program was as follows: $5-95 \% \mathrm{~B}$ from 0 tol1.5 $\mathrm{min}, 95 \% \mathrm{~B}$ from 11.5 to $13.5 \mathrm{~min}$ in positive ionization mode, and 5-95\% B from 0 tol7min/95\% B from 17 to $19 \mathrm{~min}$ in negative ionization mode. The injection volume was $2 \mu \mathrm{l}$, and the flow rate was set at $0.4 \mathrm{ml} / \mathrm{min}$.

Parameters for analysis were set using ESIin both the positive and negative ionization modes (ESI + and ESI-). Ultra-high pure helium ( $\mathrm{He}$ ) was used as the collision gas, while high purity nitrogen (N2) was used as the nebulizing gas. The parameters were set as follows: cone voltage: $40 \mathrm{~V}$; capillary voltage for ESI+: 3,000V; capillary voltage for ESI-: $2,500 \mathrm{~V}$; cone gas rate: $50 \mathrm{~L} / \mathrm{h}$; desolvation gas rate; 800 $\mathrm{L} / \mathrm{h}$, source temperature: $100^{\circ} \mathrm{C}$; mother ion collision energy: $6 \mathrm{eV}$. The data acquisition rate was $0.2 \mathrm{~s}$. The mass range was scanned from Da 100-1,200.

The raw data acquired via UPLC/Q-TOF-MS were analyzed using Marker Lynx Applications Manager version 4.1 (Waters, Manchester, UK). This allowed for data pretreatment procedures such as peak detection, deconvolution, normalization, alignment, and data reduction to give a list of mass and retention times that paired with the corresponding intensities for all detected peaks from each data file in the dataset.

2.8. Statistical Analysis. All data were expressed as the mean \pm the standard error of the mean (SEM). Statistical analyses were performed using SPSS 19.0(IBM, New York, USA). Locomotor activity data were assessed using repeatedmeasures analyses of variance (ANOVA). Sleep monitoring data were analyzed using one-way ANOVA, following which the least significant difference (LSD) method was used for comparisons among groups. In all cases, $\mathrm{P}$ values $<0.05$ were considered statistically significant.

\section{Results}

3.1. Sedative Effects of PRPCA in Mice. We evaluated locomotor activity data for each day after intragastric administration to examine the sedative effects of PRPCA in mice and determine the optimal concentration for achieving these effects. The results are shown in Tables 1, 2, and 3 and Figure 1.

Although significant differences in locomotor activity were observed among the groups $(\mathrm{F}=4.267, \mathrm{P} \leq 0.001)$ (Table 1), no such differences were observed among the different time points $(\mathrm{F}=2.133, \mathrm{P}=0.088)$. We also observed an interaction between group and treatment time $(\mathrm{F}=6.242$, $\mathrm{P}=0.004)$. At the same time point, we observed significant differences among the groups from days 8 to 14 (Table 2). 
TABLE 2: Comparison among groups at the same time point.

\begin{tabular}{cccccccc}
\hline & $\mathrm{d} 8$ & $\mathrm{~d} 9$ & $\mathrm{~d} 10$ & $\mathrm{~d} 11$ & $\mathrm{~d} 12$ & $\mathrm{~d} 13$ & $\mathrm{~d} 14$ \\
\hline $\mathrm{F}$ & 8.17 & 6.937 & 6.733 & 7.416 & 7.526 & 18.346 & 13.872 \\
$\mathrm{P}$ & $0.001^{* *}$ & $0.002^{* *}$ & $0.003^{* *}$ & $0.002^{* *}$ & $0.002^{* *}$ & $0^{* *}$ & $0^{* *}$ \\
\hline
\end{tabular}

TABLE 3: Average locomotor activity in each group (count/24h).

\begin{tabular}{lccccc}
\hline & DZP & PRPCA 1 & PRPCA 2 & PRPCA 3 & SWFI \\
\hline $\bar{X}$ & 50226.04 & 37239.75 & 48008.89 & 47746.07 & 62956.95 \\
S & 11057.37 & 9772.69 & 9638.59 & 9940.67 & 13226.20 \\
DZP & & $0.025^{* *}$ & 0.676 & 0.640 & $0.027^{* *}$ \\
SWFI & & $\leq 0.001^{\Delta \Delta}$ & $0.012^{\Delta \Delta}$ & $0.010^{\Delta \Delta}$ & \\
\hline
\end{tabular}

${ }^{* *}$ P value vs. DZP, ${ }^{\Delta \Delta}$ P value vs. SWFI. DZP: diazepam; PRPCA: Pinelliae Rhizoma Praeparatum Cum Alumine; SWFI: sterile water for injection.

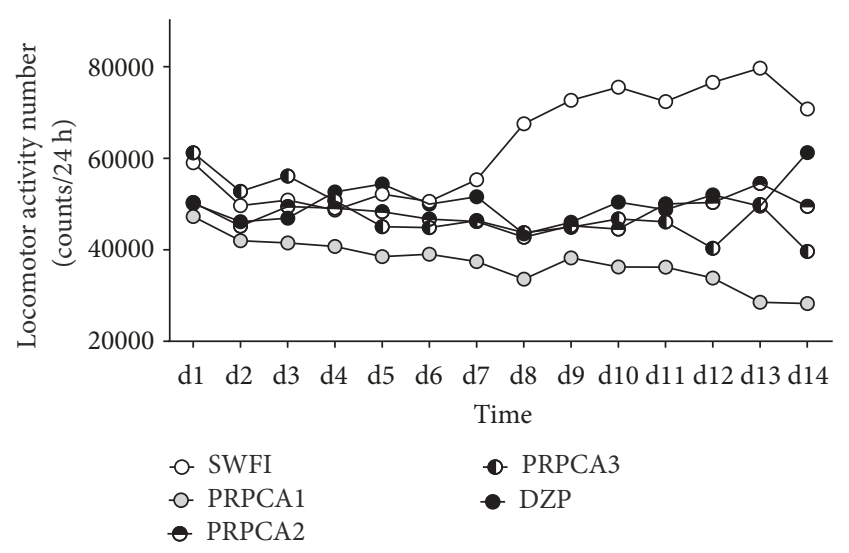

FIGURE 1: Locomotor activity following intragastric administration. White, gray, horizontal black/white, vertical black/white, and black circles represent the profiles for the SWFI, PRPCA 1, PRPCA 2, PRPCA 3, and DZP group, respectively. SWFI: sterile water for injection; PRPCA: Pinelliae Rhizoma Praeparatum Cum Alumine; DZP: diazepam.

Table 3 shows that PRPCA 1, PRPCA 2, PRPCA 3, and DZP reduced the total locomotor activity in mice, when compared with SWFI $(\mathrm{P} \leq 0.001, \mathrm{P}=0.012, \mathrm{P}=0.010$, and $\mathrm{P}=0.027$, respectively). Locomotor activity was lower in the PRPCA 1 group than in the remaining four groups from days 1 to 14 (Figure 1).

3.2. PRPCA Decreased Wakefulness and Increased Sleep in Mice. Based on amplitude and frequency results from the EEG/EMG analyses, sleep-wake stages were divided into wakefulness, NREM sleep, and REM sleep [21]. As shown in Figure 2(a), PRPCA decreased the hourly wakefulness time by $46.30 \%$ at 9:00 $\mathrm{AM}(\Delta \mathrm{P}<0.05)$ and increased the hourly NREM sleep time by 1.90-fold at 9:00 AM $(\Delta \mathrm{P}<0.05)$, when compared with SWFI. PRPCA also increased the hourly REM sleep time by 92 -fold at 4:00 AM $(* \mathrm{P}<0.05)$, relative to DZP. DZP increased the hourly NREM sleep time by 1.68 -fold at 9:00AM $\left({ }^{\#} \mathrm{P}<0.05\right)$, when compared with SWFI. Increases in NREM sleep coincided with decreases in wakefulness.

The total amount of time spent in wakefulness, NREM, and REM sleep is summarized in Figure 2(b). PRPCA increased REM sleep by 18-fold during the light phase $(* \mathrm{P}<0.05)$, relative to DZP. No other significant differences were observed.

3.3. Changes in the Number of Bouts Induced by PRPCA. To better understand the sleep-wake profile induced by PRPCA, we examined the distributions of bouts of wakefulness, NREM, and REM sleep as a function of the duration of each bout or episode (Figure 3). PRPCA decreased the number of bouts of wakefulness with durations of 16-32s and 32-64s by $38.91 \%$ and $32.68 \%\left({ }^{\Delta} \mathrm{P}<0.05,{ }^{\Delta} \mathrm{P}<0.05\right)$, respectively, and increased the number of bouts of NREM sleep with durations of $128-256$ s by 2.18 -fold $\left({ }^{\Delta} \mathrm{P}<0.05\right)$ during the dark phase, relative to SWFI. In addition, DZP decreased the number of bouts of wakefulness with durations of 16-32s and 32-64s by $35.09 \%$ and $33.33 \%\left({ }^{\Delta} \mathrm{P}<0.05,{ }^{\Delta} \mathrm{P}<0.05\right)$, respectively. However, we observed no significant changes in the transition between different sleep stages or the number of episodes for different sleep stages.

3.4. Characteristics of the Compounds in PRPCA. The identities of all detected peaks in PRPCA were determined via UPLC/Q-TOF-MS. A total of 32 compounds were identified and categorized into 19 alkaloids, three volatile oils, two fatty acids, two phenylpropanoids, and others. The representative LC-MS chromatograms are shown in Figure 4. Table 4 lists the characteristics of the 32 identified compounds.

\section{Discussion}

In the present study, we investigated the sedative effects of different concentrations of PRPCA, observing that lower concentrations of PRPCA were more effective at inducing sedation than higher concentrations, when compared with SWFI. The relatively worse sedative effects of PRPCA 2 and PRPCA 3 may be due to an inability to absorb and utilize such high concentrations of the drug. Thus, our results highlight the need for further studies regarding the most appropriate concentration of PRPCA for treatment, as even lower concentrations may be effective.

In addition, we observed that successive PRPCA administration was associated with reduced locomotor activity in 

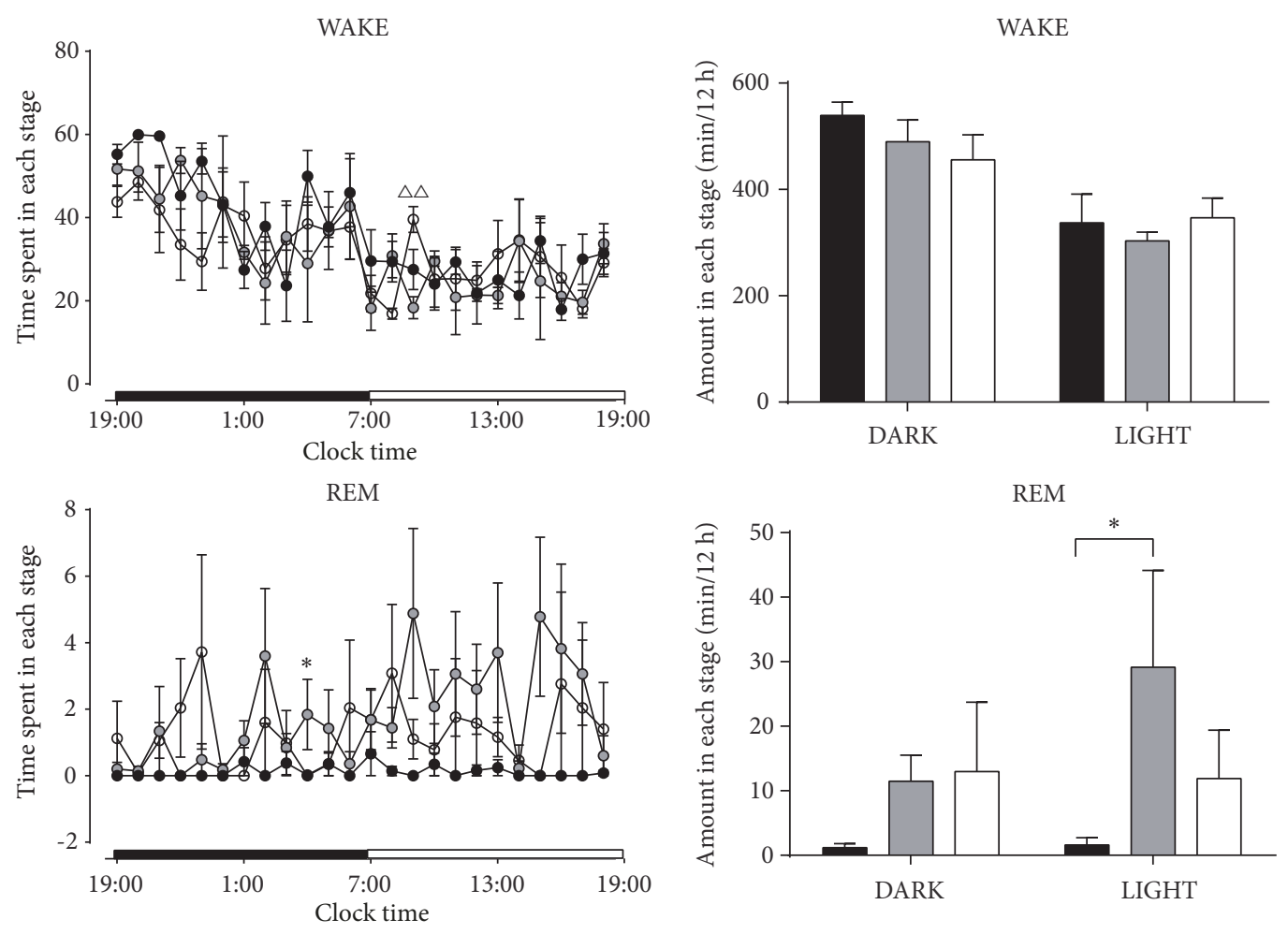

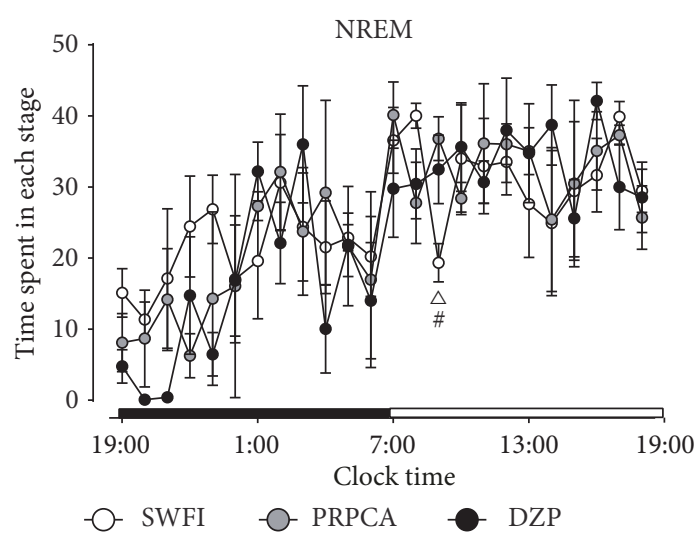

(a)

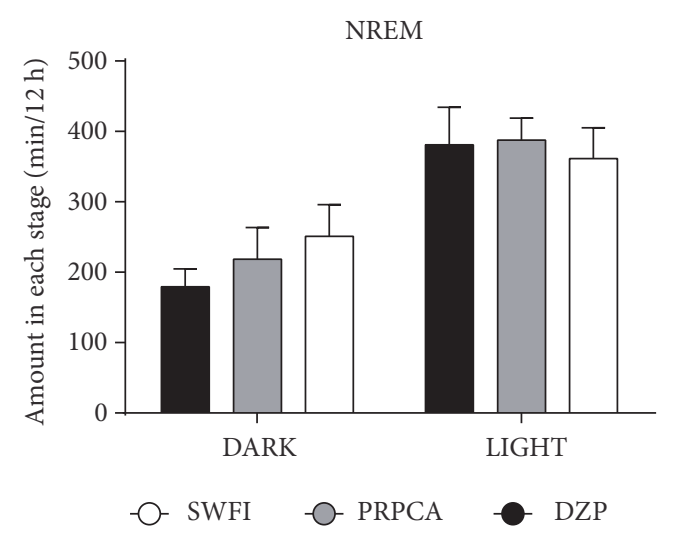

(b)

FIGURE 2: Sleep-stage distributions produced by SWFI, PRPCA, and DZP. (a) Time course of changes in wakefulness, REM sleep, and NREM sleep. Each circle represents the mean hourly amount of time spent in each stage. The horizontal open and filled bars on the X-axes indicate the 12-h light and 12-h dark periods, respectively. (b) Total time spent in wakefulness, REM sleep, and NREM sleep during the 12-h light and 12-h dark periods. White, gray, and black circles/bars represent the profiles for the SWFI, PRPCA, and DZP groups, respectively. Data are expressed as the mean $\pm \mathrm{SEM} ;{ }^{\#} \mathrm{P}<0.05$ : PRPCA vs. SWFI; $* \mathrm{P}<0.05$ : PRPCA vs. DZP; ${ }^{\Delta} \mathrm{P}<0.01$ and ${ }^{\Delta} \mathrm{P}<0.05$ : SWFI vs. DZP as assessed using ANOVA followed by the LSD test. SWFI: sterile water for injection; PRPCA: Pinelliae Rhizoma Praeparatum Cum Alumine; DZP: diazepam; REM: rapid eye movement; NREM: nonrapid eye movement; ANOVA: analysis of variance: LSD: least significant difference.

mice, suggesting a cumulative effect of PRPCA treatment. Furthermore, locomotor activity counts among the different groups at the same time differed beginning on day 8 , suggesting that PRPCA should be successively administered for more than 8 days to achieve a better curative effect. Unfortunately, we were unable to determine an unequivocal time course for treatment because the experiment was performed for a limited number of days, necessitating further studies with longer experimental periods.
We then investigated the hypnotic effects and mechanisms underlying the efficacy of PRPCA via sleep and vigilance state analyses. Our results indicated that PRPCA reduced hourly wakefulness time and increased hourly NREM sleep time at 9:00 AM (Figure 2(a)). In addition, PRPCA treatment increased the amount of 12-h REM sleep time during the light phase. Such results indicate that the hypnotic effects of PRPCA occurred in accordance with the physiological sleep-wake rhythm of mice. Further analysis 

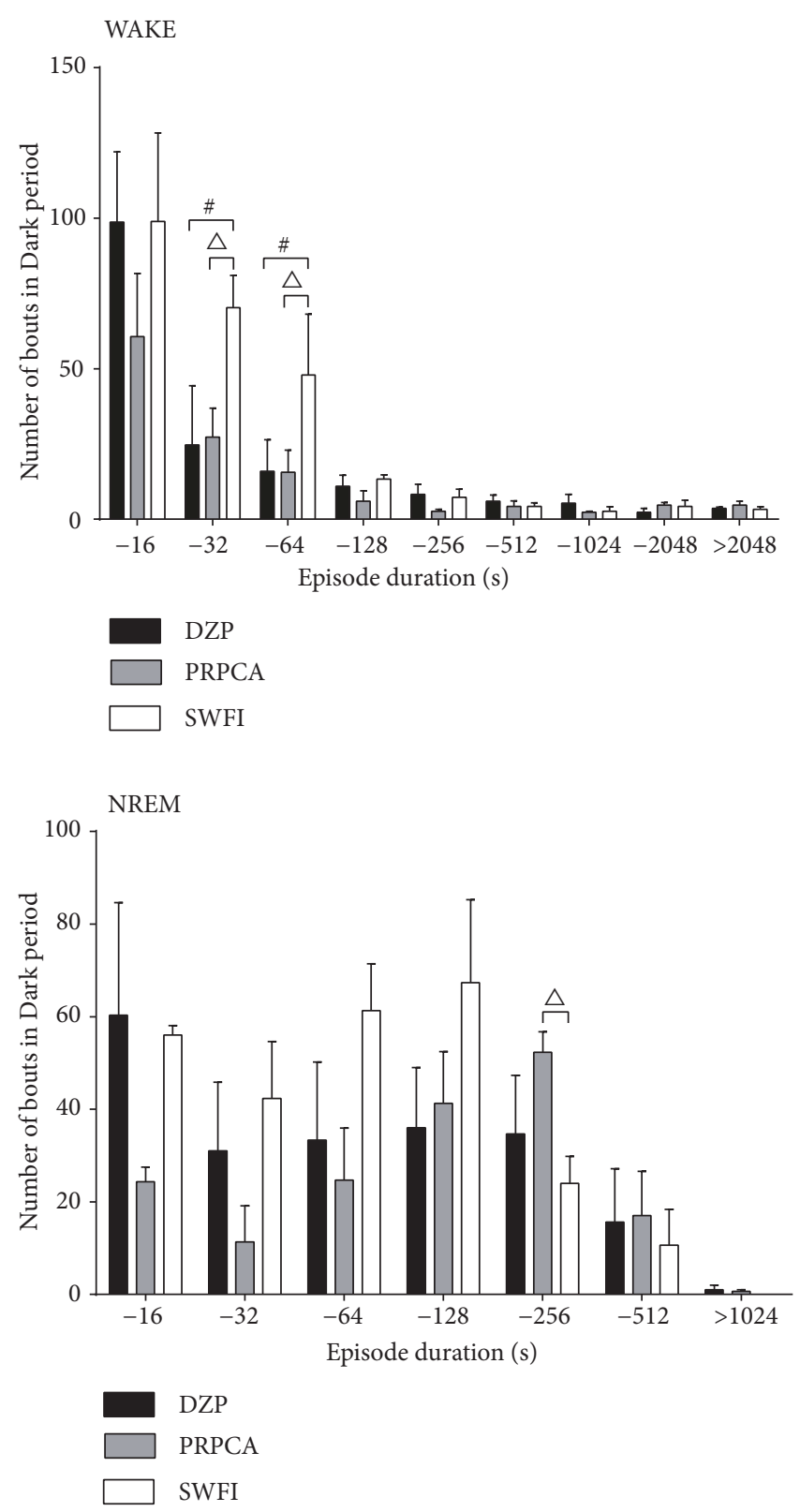

FIGURE 3: Bouts of wakefulness and NREM sleep during the dark period. White, gray, and black bars show the profiles for the SWFI, PRPCA, and DZP groups, respectively. The negative time values on the X-axis represent the following periods, respectively: 0-16 s, 16-32 s, 32-64 s, 64-128 s, 128-256 s, 256-512 s, 512-1024 s, and 1024-2048 s. Data are expressed as the mean \pm SEM; ${ }^{\#} \mathrm{P}<0.05: \mathrm{PRPCA}$ vs. SWFI; ${ }^{\Delta} \mathrm{P}<0.05$ SWFI vs. DZP as assessed using ANOVA followed by the LSD test. NREM: nonrapid eye movement; SWFI: sterile water for injection; PRPCA: Pinelliae Rhizoma Praeparatum Cum Alumine; DZP: diazepam; ANOVA: analysis of variance; LSD: least significant difference.

revealed that PRPCA treatment reduced the number of bouts of wakefulness with shorter durations (16-32s, 32-64s) and increased the number of bouts of NREM sleep with longer durations (128-256s), suggesting that PRPCA can improve sleep fragmentation and sleep quality.

In order to provide insight into the extraction of drug monomers and inform the preparation of safer, more convenient drugs, we utilized chromatography and MS to detect the main chemical components of PRPCA. We identified 32 compounds in PRPCA, including 19 alkaloids, three volatile oils, two phenylpropanoids, two fatty acids, one cerebroside, one anthraquinone, and four others. When considering relative abundance, alkaloids accounted for $32.74 \%$, fatty acids accounted for $49.73 \%$, and volatile oils accounted for $3.09 \%$ of PRPCA components. These three types of components accounted for $85.56 \%$ of the total ingredients identified. One previous study reported that $\mathrm{PR}$ is rich in alkaloids, volatile oils, fatty acids, and other ingredients, which can reduce the activity of some enzymes in tissue cells, thereby inhibiting the central nervous system and playing a role in sedation and hypnosis [22]. Such results provide a range of options for subsequent studies regarding drug monomers. 
TABLE 4: Chemical components of PRPCA.

\begin{tabular}{|c|c|c|c|c|c|c|}
\hline No. & $\operatorname{Rt}(\min )$ & $\begin{array}{c}{[\mathrm{M}+\mathrm{H}]^{+} /[\mathrm{M}-} \\
\mathrm{H}]^{-}(\mathrm{m} / \mathrm{z})^{1}\end{array}$ & Relative abundance $^{2}(\%)$ & Formula & Identification & Component type \\
\hline 1 & 0.49 & $175.1055 /$ & 0.846276 & $\mathrm{C}_{6} \mathrm{H}_{14} \mathrm{~N}_{4} \mathrm{O}_{2}$ & L-arginine & Alkaloid \\
\hline 2 & 0.51 & $134.0433 /$ & 1.543210 & $\mathrm{C}_{4} \mathrm{H}_{7} \mathrm{NO}_{4}$ & Aspartic acid & Alkaloid \\
\hline 3 & 0.53 & $173.2322 /$ & 20.549580 & $\mathrm{C}_{9} \mathrm{H}_{16} \mathrm{O}_{3}$ & 9-Oxo-nonanoic acid & Fatty acid \\
\hline 4 & 0.53 & $104.0692 /$ & 1.931501 & $\mathrm{C}_{4} \mathrm{H}_{9} \mathrm{NO}_{2}$ & r-Aminobutyric acid & Alkaloid \\
\hline 5 & 0.55 & $90.0647 /$ & 1.324170 & $\mathrm{C}_{3} \mathrm{H}_{7} \mathrm{NO}_{2}$ & Alanine & Alkaloid \\
\hline 6 & 0.55 & $116.0694 /$ & 0.667065 & $\mathrm{C}_{5} \mathrm{H}_{9} \mathrm{NO}_{2}$ & Proline & Alkaloid \\
\hline 7 & $\begin{array}{c}0.56 / \\
0.56\end{array}$ & $\begin{array}{c}343.1393 / \\
341.1121\end{array}$ & $\begin{array}{c}17.502990 / \\
9.261898\end{array}$ & $\mathrm{C}_{16} \mathrm{H}_{22} \mathrm{O}_{8}$ & Coniferin & Phenylpropanoid \\
\hline 8 & 0.56 & $127.0395 /$ & 0.696933 & $\mathrm{C}_{6} \mathrm{H}_{6} \mathrm{O}_{3}$ & 5-Hydroxymethylfurfural & Alkaloid \\
\hline 9 & 0.56 & $127.0395 /$ & 0.527680 & $\mathrm{C}_{5} \mathrm{H}_{6} \mathrm{~N}_{2} \mathrm{O}_{2}$ & 5-Methyl uracil & Alkaloid \\
\hline 10 & 0.56 & $181.0703 /$ & 1.971326 & $\mathrm{C}_{9} \mathrm{H}_{8} \mathrm{O}_{4}$ & Caffeic acid & Other \\
\hline 11 & $\begin{array}{c}0.56 / \\
0.55\end{array}$ & $\begin{array}{l}145.0491 / \\
143.0344\end{array}$ & $\begin{array}{l}2.031063 \\
/ 1.082930\end{array}$ & $\mathrm{C}_{6} \mathrm{H}_{8} \mathrm{O}_{4}$ & 1,6:3,4-Dianhydro- $\beta$-D-allosep & Other \\
\hline 12 & $\begin{array}{c}0.56 / \\
0.56\end{array}$ & $\begin{array}{c}343.122 / \\
341.122\end{array}$ & $\begin{array}{l}17.562720 \\
/ 9.233400\end{array}$ & $\mathrm{C}_{12} \mathrm{H}_{22} \mathrm{O}_{11}$ & Sucrose & Phenylpropanoid \\
\hline 13 & 0.57 & $148.0641 /$ & 0.975707 & $\mathrm{C}_{5} \mathrm{H}_{9} \mathrm{NO}_{4}$ & Glutamic acid & Alkaloid \\
\hline 14 & 0.60 & $118.0849 /$ & 2.538829 & $\mathrm{C}_{5} \mathrm{H}_{11} \mathrm{NO}_{2}$ & Valine & Alkaloid \\
\hline 15 & 0.68 & $137.0473 /$ & 1.513341 & $\mathrm{C}_{5} \mathrm{H}_{4} \mathrm{~N}_{4} \mathrm{O}$ & 6 - purine & Alkaloid \\
\hline 16 & 0.69 & $182.0808 /$ & 1.184787 & $\mathrm{C}_{9} \mathrm{H}_{11} \mathrm{NO}_{3}$ & Tyrosine & Alkaloid \\
\hline 17 & 0.69 & $150.0623 /$ & 0.796495 & $\mathrm{C}_{5} \mathrm{H}_{11} \mathrm{O}_{2} \mathrm{NS}$ & Methionine & Alkaloid \\
\hline 18 & 0.71 & 132.101/ & 2.489048 & $\mathrm{C}_{6} \mathrm{H}_{13} \mathrm{NO}_{2}$ & Isoleucine & Alkaloid \\
\hline 19 & 0.71 & $136.0645 /$ & 3.803266 & $\mathrm{C}_{5} \mathrm{H}_{5} \mathrm{~N}_{5}$ & Pedatisectine B & Alkaloid \\
\hline 20 & 1.28 & $166.0844 /$ & 6.800080 & $\mathrm{C}_{9} \mathrm{H}_{11} \mathrm{NO}_{2}$ & Phenylalanine & Alkaloid \\
\hline 21 & 1.29 & $120.0781 /$ & 1.324174 & $\mathrm{C}_{4} \mathrm{H}_{9} \mathrm{NO}_{3}$ & Threonine & Alkaloid \\
\hline 22 & 1.30 & $167.0919 /$ & 0.527678 & $\mathrm{C}_{10} \mathrm{H}_{14} \mathrm{O}_{2}$ & 5-Amyl-2-pyrone & Volatile oil \\
\hline 23 & 1.30 & $268.1042 /$ & 2.220231 & $\mathrm{C}_{10} \mathrm{H}_{13} \mathrm{~N}_{5} \mathrm{O}_{4}$ & Adenosine & Alkaloid \\
\hline 24 & $\begin{array}{l}1.50 / \\
4.06\end{array}$ & $\begin{array}{l}263.1381 / \\
261.1375\end{array}$ & $\begin{array}{c}1.702509 / \\
1.738387\end{array}$ & $\mathrm{C}_{14} \mathrm{H}_{18} \mathrm{~N}_{2} \mathrm{O}_{3}$ & Cyclo-(Val-L-Tyr) & Alkaloid \\
\hline 25 & 3.35 & $696.538 /$ & 1.403823 & $\mathrm{C}_{40} \mathrm{H}_{73} \mathrm{NO}_{8}$ & $\begin{array}{l}\text { 1-O-glucosyl-N-2 }{ }^{\prime} \text {-palmitoyl-4,8- } \\
\text { sphingodienine }\end{array}$ & Cerebroside \\
\hline 26 & 3.59 & $97.0749 /$ & 1.652728 & $\mathrm{C}_{6} \mathrm{H}_{8} \mathrm{O}$ & 2-Ethenyl butenal & Volatile oil \\
\hline 27 & /3.68 & $/ 167.0421$ & 1.766885 & $\mathrm{C}_{8} \mathrm{H}_{8} \mathrm{O}_{4}$ & Vanillic acid & Other \\
\hline 28 & $\begin{array}{l}4.18 / \\
5.32\end{array}$ & $\begin{array}{l}255.0653 / \\
253.0549\end{array}$ & $\begin{array}{l}2.359618 \\
/ 4.502707\end{array}$ & $\mathrm{C}_{15} \mathrm{H}_{10} \mathrm{O}_{4}$ & Chrysophanol & Anthraquinone \\
\hline 29 & 5.51 & /191.0798 & 0.911941 & $\mathrm{C}_{15} \mathrm{H}_{12}$ & Methyl phenanthrene & Volatile oil \\
\hline 30 & 5.72 & $269.0709 /$ & 1.553166 & $\mathrm{C}_{10} \mathrm{H}_{12} \mathrm{~N}_{4} \mathrm{O}_{5}$ & Inosine & Alkaloid \\
\hline 31 & /7.11 & /329.2371 & 29.182100 & $\mathrm{C}_{18} \mathrm{H}_{34} \mathrm{O}_{5}$ & Pinellic acid & Fatty acid \\
\hline 32 & $/ 14.05$ & $/ 293.1834$ & 40.609860 & $\mathrm{C}_{17} \mathrm{H}_{26} \mathrm{O}_{4}$ & Gingerol & Other \\
\hline
\end{tabular}

Note 1: Data to the left of " " represent the $\mathrm{m} / \mathrm{z}$ of $[\mathrm{M}+\mathrm{H}]$. Data to the right of " " represent the $\mathrm{m} / \mathrm{z}$ of $[\mathrm{M}-\mathrm{H}]$. Note 2: Relative abundance values were obtained via normalization.

However, the present study possesses some limitations of note. First, further studies involving longer experimental periods are required to determine the most appropriate concentration and unequivocal time course for treatment. Second, although we demonstrated that PRPCA can promote sleep by reducing wakefulness and increasing sleep time, the underlying physiological principles remain to be elucidated. Third, the roles of the 32 identified PRPCA components in promoting sleep must be investigated in future studies.

\section{Conclusion}

Our findings demonstrated that PRPCA exerts cumulative sedative and hypnotic effects. PRPCA improved sleep quality by increasing REM sleep and reducing sleep fragmentation in accordance with the physiological sleep-wake rhythm of mice, suggesting that PRPCA can be used in the clinical treatment of insomnia. Although alkaloids and fatty acids appear to play a major role, the mechanisms underlying the 


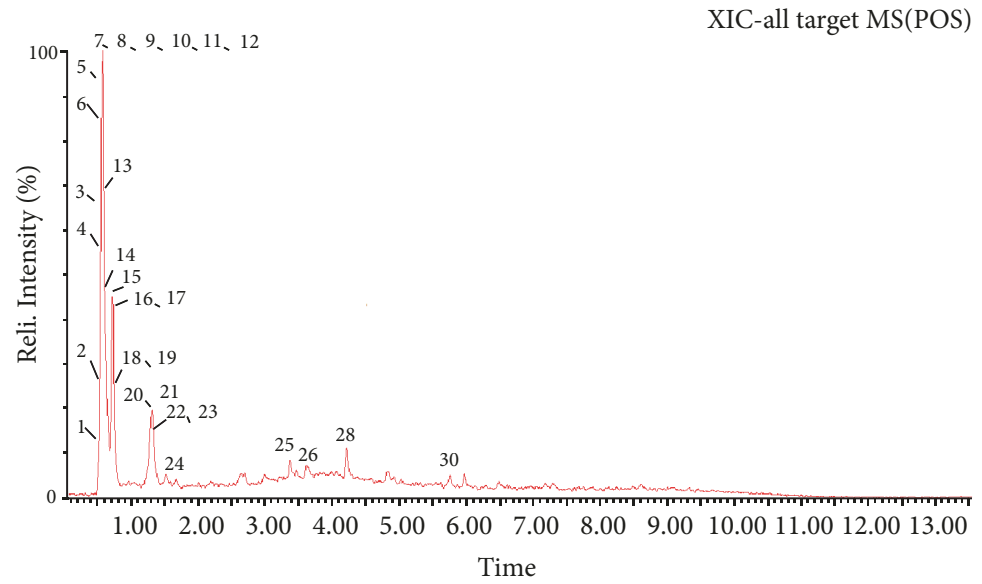

(a)

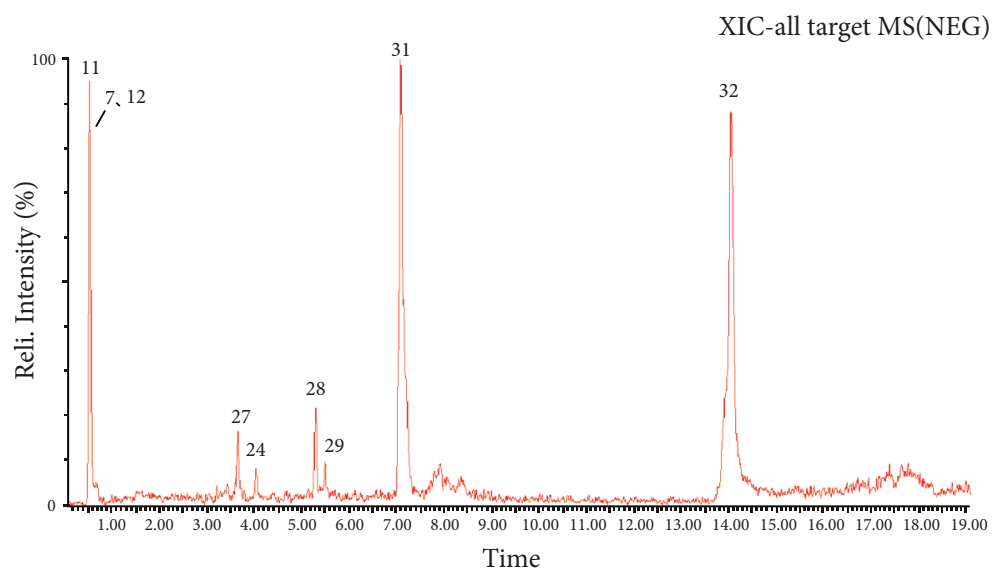

(b)

FIGURE 4: Extracted ion chromatogram of compounds in PRPCA identified via UPLC/Q-TOF-ESI/MS. (a) Extracted ion chromatogram in positive mode (ESI+). (b) Extracted ion chromatogram in negative mode (ESI-). PRPCA: Pinelliae Rhizoma Praeparatum Cum Alumine; UPLC: ultra-performance liquid chromatography; Q-TOF: quadrupole time-of-flight; ESI: electrospray ionization; MS: mass spectrometry.

sedative and hypnotic effects of PRPCA remain to be fully elucidated.

\section{Data Availability}

The data used to support the findings of this study are available from the corresponding author upon request.

\section{Conflicts of Interest}

The authors declare that there are no potential conflicts of interest with respect to the research, authorship, and/or publication of this article.

\section{Authors' Contributions}

Sisi Lin and Bo Nie contributed equally to this work.

\section{Acknowledgments}

This study was supported by the Department of Pharmacology, School of Basic Medical Sciences, Fudan University, Shanghai, China, the National Natural Science Foundation of China [Grant number 81673733], and the Outstanding Young Talent Fund Project of Chinese Medicine of Zhejiang Province, China [Grant number 2011zq014].

\section{References}

[1] D. Burman, "Sleep disorders: insomnia," FP Essentials, vol. 460, pp. 22-28, 2017.

[2] D. Léger and V. Bayon, "Societal costs of insomnia," Sleep Medicine Reviews, vol. 14, no. 6, pp. 379-389, 2010.

[3] R. C. Kessler, P. A. Berglund, C. Coulouvrat et al., "Insomnia and the performances of US workers: results from the America insomnia survey," Sleep, vol. 34, no. 9, pp. 1161-1171, 2011. 
[4] E. S. Zhou, P. Gardiner, and S. M. Bertisch, "Integrative medicine for insomnia," Medical Clinics of North America, vol. 101, no. 5, pp. 865-879, 2017.

[5] A. Thomas, M. Grandner, S. Nowakowski, G. Nesom, C. Corbitt, and M. L. Perlis, "Where are the behavioral sleep medicine providers and where are they needed? a geographic assessment," Behavioral Sleep Medicine, vol. 14, no. 6, pp. 1-12, 2016.

[6] M. L. Perlis and M. T. Smith, "How can we make CBT-I and other BSM services widely available?" Journal of Clinical Sleep Medicine, vol. 4, no. 1, pp. 11-13, 2008.

[7] W. R. Pigeon, V. M. Crabtree, and M. R. Scherer, "The future of behavioral sleep medicine," Journal of Clinical Sleep Medicine, vol. 3, no. 1, pp. 73-79, 2007.

[8] G. Y. Cai and L. Y. Huang, "Application of Pinelliae ternata in the treatment of insomnia," Jilin Journal of Chinese Medicine, vol. 37, no. 7, pp. 729-731, 2017.

[9] Chinese Pharmacopoeia Commission, Chinese Crude Drug and Cut Crude Drug in Pharmacopoeia of the People's Republic of China, vol. 1, China Medical Science Press, Beijing, China, 2015.

[10] Y. M. Song, L. R. Cui, and J. W. Li, "Literature analysis of ancient insomnia prescription," Journal of Shandong University of Traditional Chinese Medicine, vol. 36, no. 5, pp. 392-394, 2012.

[11] S. Lin, B. Nie, G. Yao, H. Yang, R. Ye, and Z. Yuan, "Pinellia ternata (Thunb.) Makino Preparation promotes sleep by increasing REM sleep," Natural Product Research (Formerly Natural Product Letters), pp. 1-4, 2018.

[12] W. T. Tao, H. L. Yu, H. Wu et al., "Irritation and fingerprint of pinelliae rhizome, unprocessed, processed with ethanol and processed with alum," Chinese Traditional Patent Medicine, vol. 34, pp. 899-904, 2013.

[13] M. M. Fang, S. S. Lin, and Z. Z. Yuan, "Comparison of different processed products of Rhrizoma Pinelliae sedative effect on mice," Journal of Medicine Research, vol. 45, no. 2, pp. 71-75, 2016.

[14] M. Miranda-Anaya, D. Luna-Moreno, A. Carmona-Castro, and M. Díaz-Muñoz, "Differences in photic entrainment of circadian locomotor activity between lean and obese volcano mice (Neotomodon alstoni)," Journal of Circadian Rhythms, vol. 15, no. 1, 2017.

[15] L. Chen, D. Yin, T.-X. Wang et al., "Basal forebrain cholinergic neurons primarily contribute to inhibition of electroencephalogram delta activity, rather than inducing behavioral wakefulness in mice," Neuropsychopharmacology, vol. 41, no. 8, pp. 21332146, 2016.

[16] K. B. J. Franklin and G. Paxinos, The Mouse Brain in Stereotaxic Coordinates, Compact Second Edition, Elsevier Academic Press, San Diego, Calif, USA, 2012.

[17] M. Y. Um, S. Kim, Y.-H. Jin et al., "A novel neurological function of rice bran: a standardized rice bran supplement promotes non-rapid eye movement sleep in mice through histamine $\mathrm{H} 1$ receptors," Molecular Nutrition \& Food Research, vol. 61, no. 11, 2017.

[18] M.-Q. Zhang, T.-X. Wang, R. Li et al., "Helicid alleviates pain and sleep disturbances in a neuropathic pain-like model in mice," Journal of Sleep Research, vol. 26, no. 3, pp. 386-393, 2017.

[19] Z. Zhang, H. J. Wang, D. R. Wang et al., "Red light at intensities above 10lx alters sleep-wake behavior in mice," Light- Science \& Applications, vol. 6, no. 5, Article ID e16231, 2017.

[20] Q. Wang, X.-F. Yue, W.-M. Qu et al., "Morphine inhibits sleeppromoting neurons in the ventrolateral preoptic area via mu receptors and induces wakefulness in rats," Neuropsychopharmacology, vol. 38, no. 5, pp. 791-801, 2013.
[21] C. R. Chen, X. Z. Zhou, Y. J. Luo et al., “a major bioactive constituent of the bark of Magnolia officinalis, induces sleep via the benzodiazepine site of GABA (A) receptor in mice," Neuropharmacology, vol. 63, no. 6, pp. 1191-1199, 2012.

[22] D. M. Wang, "Observation and analysis of clinical mechanism of pinellia ternata treating insomnia," Nei Mongol Journal of Traditional Chinese Medicine, vol. 33, no. 5, p. 17, 2014. 


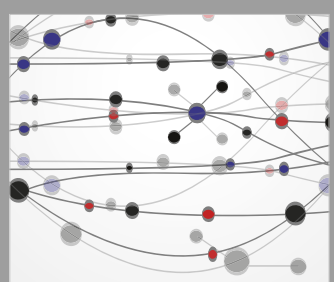

The Scientific World Journal
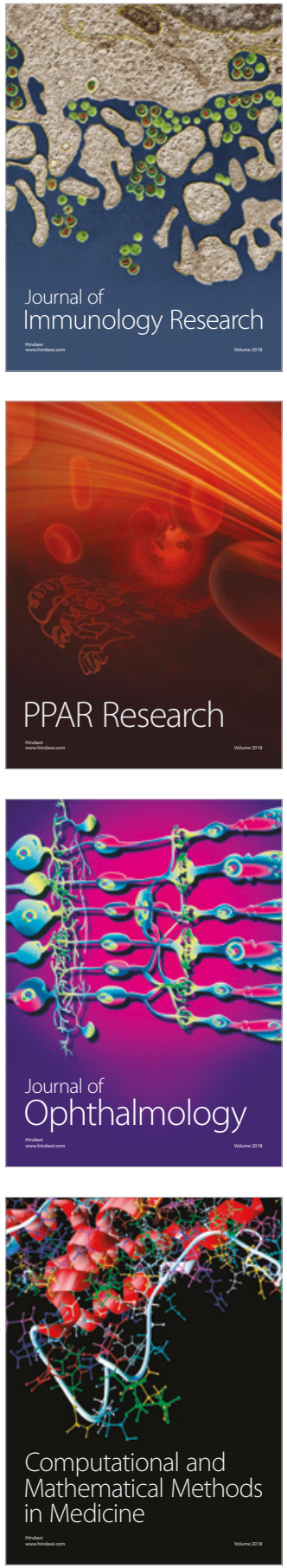

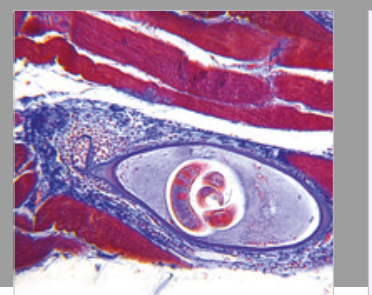

Gastroenterology Research and Practice

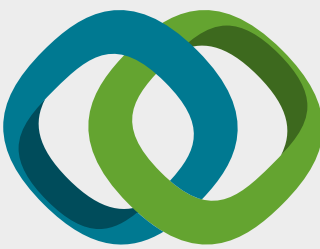

\section{Hindawi}

Submit your manuscripts at

www.hindawi.com
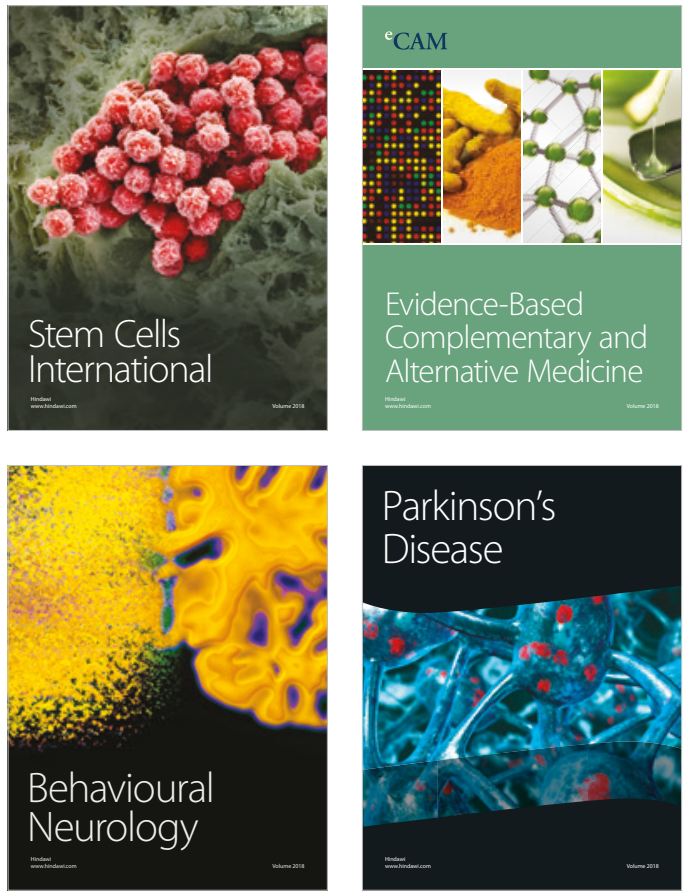

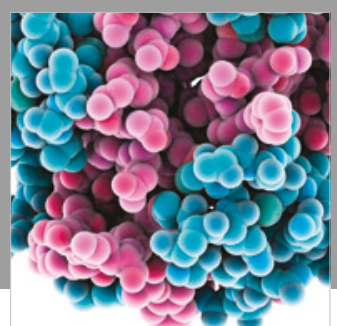

ournal of

Diabetes Research

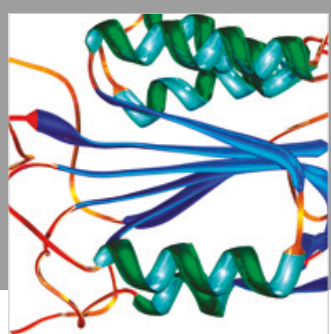

Disease Markers
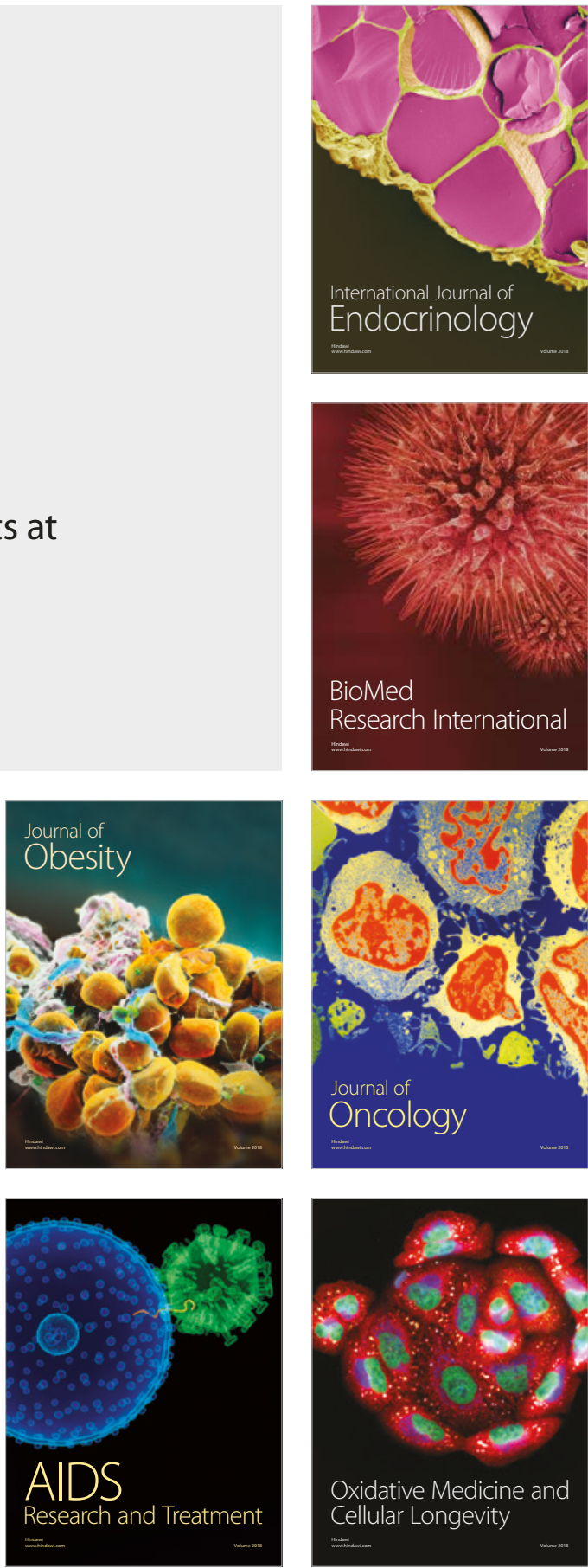